\title{
Procedimenti narrativi nei romanzi di Giovan Ambrosio Marini
}

Se nella Francia dell'età barocca il romanzo fu al centro di discussioni vivaci il cui interesse teorico, al di là dei casi di partenza, si estese ai procedimenti narrativi tipici del genere letterario, con esiti (si pensi a un Valincour, ad un Sorel) di singolare modernità, e se, ben oltre il campo delle polemiche occasionali, un'opera come la Lettre o Traité de l'origine des romans di Pierre Daniel Huet, decretando l'esistenza metastorica e universale di quel tipo di letteratura, adottava per esso la normativa epica e opponeva di fatto una credenziale genealogica e aristotelica alla giustificazione per la moda, se di tutto questo, dunque, si dibatteva nella cultura francese, in Italia al contrario la rimozione del problema romanzesco fu, al livello più culto della teoresi, pressoché totale, lasciando spazio a innumerevoli giudizi sommari e a scandalizzate condanne.

Del romanzo, invero si parlò molto, ma più come del prodotto della moda e della decadenza dei costumi letterari che come oggetto da approfondire e su cui teorizzare. ${ }^{1}$ Mentre Huet, nella censura severa delle idee di Giraldi e Pigna sulla questione dell'unità della favola e nella prescrizione assoluta del verisimile, del conveniente, del decorum, sembrava collegarsi alle tematiche cinquecentesche della prima polemica sul Furioso e della successiva disputa su Ariosto e Tasso, in Italia il problema della regolarità del romanzo, sdegnato dai trattatisti, fu variamente affrontato in un colloquio diretto tra romanzieri e lettori. Il luogo di questo discorso era nelle pagine di presentazione, sovente polemiche, premesse ai romanzi. ${ }^{2}$ In esse, pur tra proclamazioni spesso disattese, l'autore (non 'implicito' ma parlante, nella zona liminare del testo, al di qua del narrato) si rivolge al lettore mostrando una conoscenza precisa dei suoi gusti e delle sue attese, rinviando, ben oltre la cultura teorica, ad una competenza comune a produttore e ricettore, ovvero al riconoscimento delle regole del genere e alla possibilità di godere di capacità decifratorie che il testo lusinga. Di queste ricognizioni metanarrative esempi eccellenti ci offrono le premesse alle opere di Giovan Ambrosio Marini, il più ammirato, stampato e tradotto tra i romanzieri del secolo decimosettimo. ${ }^{3}$ Quelle pagine ci mostrano l'autore alle prese con la definizione di un interesse narrativo che distingue i suoi romanzi da molti prodotti coevi.

Subito appare evidente, tra le altre cose, il secco rifiuto tanto della moda del mascheramento storiografico della narrazione (dove la presunta historia era un salvacondotto per spacciare l'inverisimile), ${ }^{4}$ quanto di quella narrativa che, autodefinendosi historica, riduceva la storia a un illimitato registro 
topico in cui trascegliere motivi e intrighi legandoli secondo un'orditura non ristretta dalle norme dell'epica e appena ombreggiata di verisimile. Si veda come in queste pagine il gioco provocatorio di Marini finisca per far dubitare dell'esistenza di ogni discrimine tra veridicità storiografica e finzione romanzesca:

Qualche altro bell'humore, professandosi sol leggitore di storie, non di fole, dicevami esser ben fatto intitolare il libro, storia per avventura di Trabisonda, e nella lettera a' lettori dar loro a intendere, che tutti gli avvenimenti del Calloandro fosser casi seguiti, e quindi più volonterosamente dover essere letti costantemente affermava. A Dio non piaccia, o lettore, che sì scioccamente io ti burli. Se tu sei alcun di costoro, cui basti il dirti esser veri avvenimenti per crederli, o cui il credere che sian finti, tolga il diletto del leggerli, serioso troppo sei tu, troppo sei credulo, per te non ho scritto. Appigliati pure al Guicciardino, o a Plinio, o pure all'Alcorano, e quivi a bastalena satiati di credere, tutto è scritto per verissimo. Ciò sol vuol dirti, che al mio paese, il dire storie e favole è tutt'uno, e che ormai per tutto eguale all'una, e all'altra, si dà fede. Lo stesso, a mio credere, inferir volle il gran Boccaccio, quando nel proemio del Decamerone dice: intendo di raccontare cento novelle, o favole, o parabole, o storie. $(\text { C. Sm. })^{5}$

All'esclusione della finta historia si accompagna l'indifferenza per l'osservanza del verisimile (da molti autori dichiarata a prescindere dall'effettiva realizzazione nelle opere). Ancora una volta si demistificano le posizioni e la coerenza dei critici ostili mentre il richiamo al nome di Ariosto, pur per altri fini, non può non ricordare una tradizione tanto legittimante quanto però avversata dagli oltranzisti della regolarità epica:" "verso questi tali [i critici] ben posso dire con l'Ariosto nel Furioso che 'l lor litigio è un zero" (C. $S m$.). D'altra parte, per Marini, le "vere regole dell'arte", $\mathrm{i}$ "precetti, che alla fine sono positivi", devono cedere, nell'ordine delle priorità romanzesche, al "dilettevole, che può chiamarsi precetto naturale" (ibidem). Tale fine edonistico è talvolta limitato a sé ( $G$., premessa "a chi legge"), talaltra connesso con l'utilità ricavabile dall'ammirazione di un modello eroico perfetto, ${ }^{8}$ infine giustificato, nell'ultimo romanzo, gli Scherzi di Fortuna (l'unico di ambientazione cristiana), come il dato obbiettivo che, ai fini dell'edificazione morale, rende il mezzo romanzesco, in quanto più appetibile, più efficace dell'opera di genere devoto (cfr. la prefazione a $S$.).

Se dunque ai "precetti positivi" e alle "regole dell'arte" Marini accordava generalmente poco credito, uno tra essi tuttavia, quello dell'unità e della coerenza della favola, è professato con forza e decisione nelle premesse alle varie stesure del Calloandro, il più lungo e pretenzioso tra i suoi romanzi:

Ben posi ogni mio studio per rappresentarti una Historia Eroica, ripiena d'avvenimenti uniti sì che un corpo solo ne venga a formarsi. (C.Sc.)

Ancora, nella parte in forma dialogica della prefazione al Calloandro "sconosciuto", uno dei due interlocutori censura sia la diffusa pratica di inserire 
nell'opera digressioni novellistiche e racconti episodici secondari "raccolti da qualche novella del Boccaccio, dalle commedie di Lopez, o involato da alcun Greco poco domestico" (C.Sc.), sia l'eventuale incapacità di controllo della solidità strutturale da parte dell'autore. Nell'ultimo Calloandro, l'autore lamenta il fatto che, per l'edizione "sconosciuta", gli stampatori (i Turrini di Venezia), spacciarono la seconda parte del romanzo per componimento nuovo:

Vedi se inventione più pregiudicante al romanzo potea mai rintracciarsi. Il pregio di sì fatti componimenti, massime di macchina grande, come questo, principalmente risulta dall'unione della favola; e dove io per mantenere cotale unione mi lambicco il cervello, essi con titoli nuovi la rappresentano disunita con presentare al lettore in forma di due gemelli quel parto, che dall'esser unico, il maggior pregio raccoglie. (C. F.)

La prescrizione dell'unità coerente dalla trattatistica di matrice oraziana e aristotelica era passata nella precettistica del romanzo: Huet critica Giraldi Cinzio e Pigna per aver lodato nel romanzo cavalleresco la moltiplicazione delle azioni "senz'ordine, senza nesso, senz'arte" (32); il romanziere italiano Giovan Battista Manzini, nell'avvertenza al lettore del suo Cretideo, prescrive che il nuovo tipo di componimento debba soggiacere agli "obblighi dell'unità e dell'austerità della tessitura" dell'epica rinunciando per contro all'artificio nobilitante dell'elocuzione ornata. In Marini tuttavia l'insistenza sull'unità più che da soggezione alle regole dell'epopea, sembra originarsi dagli esiti di una competenza narrativa ben attenta e partecipe dell'impegno e delle capacità decifratorie del lettore. Si veda quanto scrive nella prefazione all'edizione "sconosciuta" del Calloandro:

... prima ch'egli t'abbia descritto l'alba nascente la chiarezza e il fosco del nuovo giorno, l'armi de' Cavalieri, la divisa, le impresse dello scudo, gli abbigliamenti de' destrieri, il manto, il moto, l'agilità, e tutto con ispiritelli e concetti per lo più divelti con le tenaglie dal concavo della Luna, onde ben spesso addiviene che conviene, perché intanto il sol tramonta, differir la pugna ad altro giorno. Prima che il Piloto habbia levate le ancore, e fatto vela, saresti giunto da Lisbona per fine alle Indie. Ma se poi per mala sorte l'Infelice Nave incontra per cammino qualche tempesta, e qui ti voglio, più naufrago sei tu che quello però che Fortuna forse ancora in porto ridurrà; ma tu sei ben sicuro che la memoria di coloro, che se vi andavano, e della cagione per cui vi andavano, in te per la dimenticanza sia naufragata. (C.Sc.)

Si può osservare come la parodia qui abbozzata, operando ciò che Šklovskij chiamava il "denudamento del procedimento" (207 e passim), enuclei due problemi essenziali legati alla temporalità narrativa: il rallentamento causato dall'amplificazione retorica, e quello prodotto dall'inserimento di descrizioni: aspetti, in fondo, di un'unica questione, essendo l'ekphrasis il luogo privilegiato della proliferazione dei tropi e dei concetti. Oggettivando e dunque forzando con l'iperbole parodistica la confrontabilità dei due orizzonti temporali della narrazione ("tempo della storia" e "tempo nel racconto", nelle 
formule di Genette, Figure III 81), e occultando la loro natura convenzionale, Marini mette in rilievo la questione dei procedimenti specifici della narrativa.

Le pagine seguenti cercheranno di individuare se e con quali strumenti l'autore traduca operativamente i principi e i precetti che si son venuti evidenziando, e in primo luogo se si segnalino strategie e artifici miranti alla costruzione di un racconto unitario e coerente nonostante la ricchezza della trama. Non interesserà a questo punto proporre modelli generali di meccanismi narrativi o patterns funzionali cui ridurre per astrazione tutti i prodotti romanzeschi del secolo. È sufficiente ricordare che ricerche condotte su altri autori hanno messo in luce schemi testuali di tipo diverso in cui il problema dell'unità coerente tanto caro a Marini è del tutto eluso, tanto nei programmi quanto nei risultati testuali. ${ }^{9} \mathrm{Si}$ tratterà piuttosto di affrontare, con strumenti narratologici meno desueti e più adatti ad un'analisi individualizzante, le opere di un romanziere di primo piano nel panorama secentesco, le uniche sopravvissute nel favore dei lettori ben oltre i limiti del secolo che le produsse. $^{10}$

\section{Procedimenti per l'unità}

La ricerca dell'unità condotta da Marini parte da un primo livello di costruzione fabulare. ${ }^{11}$ Il numero dei personaggi che agiscono in modo duraturo nel campo del racconto principale (a voce di "autore"-narratore) ${ }^{12}$ è estremamente esiguo e fortemente gerarchizzato: ${ }^{13}$ nel Calloandro, ad esempio, si riduce alle due coppie genitore-figlio (Poliarte e Calloandro; Tigrinda e Leonilda), a Acomate e Uranio (personaggi gregari e antagonisti temporanei), a Safar (meno frequentemente), a Brandilone e Chrisanta (gli antagonisti veri e propri degli eroi); negli Scherzi troviamo Cleonte e Ramira (la coppia amante) e la duchessa d'Ampuria (antagonista); nelle Gare Formidauro, Radamantero, Frontelmo, Fidalma, in modo meno gerarchizzato e con funzione meno distinta che negli altri romanzi. Quello che conta, infatti, soprattutto per i due romanzi più lunghi (Calloandro e Scherzi), non è tanto il numero degli eroi, quanto la loro gerarchicità, il loro tipo di rapporto, la dislocazione spaziale delle loro operazioni funzionali. Essi si spostano con frequenza e su distanze molto lunghe, ma la "rapidità" di questi spostamenti, e la casuale o fatale riunione negli stessi luoghi rendono poco rilevante la loro dispersione. Il continuo mascheramento e le fittizie identità dei personaggi simulano la distanza tra soggetto e oggetto della quête, rendendo inutile il loro allontanamento ed una separazione concretamente spaziale. Così come quello dei "ricercatori", anche il numero dei personaggi "ricercati" è limitato, e sostanzialmente riducibile, nel lungo Calloandro, alla coppia protagonista e, ovviamente, ai personaggi fittizi cui danno vita mascherandosi. Le "collaborazioni" nella stessa azione sono numerose, sempre motivate con l'offerta di servizio cavalleresco più che con il comune interesse, salvo poi ribaltare le situazioni quando l'oggetto si rivela, al collaboratore, essere quello della sua stessa 
quête. Così Calloandro aiuta Uranio a conquistare l'amata Mattamira, che si scopre essere Leonilda; e, negli Scherzi, Cleonte aiuta Zaido ad ottenere una certa Olinda di cui è innamorato, e che è in realtà la Ramira amata da Cleonte. Si tratta, evidentemente, di paradossali estremi di cavalleria che hanno tra $\mathrm{i}$ loro modelli la vicenda di Leone e Ruggiero nell'Orlando Furioso (Ruggiero però era consapevole fin dall'inizio dell'impresa). L'inventio mariniana è in questo modo estremamente economica, e sfrutta fino in fondo le potenzialità automoltiplicative dei singoli personaggi, soprattutto col gioco del mascheramento. Ad ogni modo va precisato che la tipologia delle inchieste varia considerevolmente da romanzo a romanzo, anche in rapporto alla coerenza e alla tenacia degli eroi. Negli Scherzi, infatti, la struttura delle peripezie è resa meno complicata tanto dalla sostanziale fissità ed unicità dell'eroe, che non ha "doppi" e non si sdoppia, quanto dalla tenacia con cui persegue la sua inchiesta, diversamente da Calloandro che cerca Leonilda in modo molto discontinuo, alternando progetti di morte, di fuga, di romitaggio, di smarrimento e momenti di attività o di piena accettazione di un non chiaro destino. Bisogna inoltre aggiungere che raramente un personaggio secondario si impegna in un'azione con obbiettivo autonomo ed eccentrico rispetto all'azione principale. La concentrazione spaziale dei personaggi dovuta al loro ritrovarsi insieme pur se in luoghi molto diversi, il coinvolgimento in azioni convergenti (collaborazioni) o antagonistiche, le disgiunzioni talvolta solo apparenti, permettono alla voce narrante di tenere sostanzialmente fissa o poco mobile la sua visuale su un solo personaggio (o su un ristretto gruppo) e su un insieme di fattori di "unità", consentono l'uso di un procedimento narrativo semplicissimo, che volentieri diremmo "a gradini" se ciò non interferisse con i molteplici usi che questa definizione ha avuto soprattutto nella tradizione critica russa (Šklovskij 37; Tomaševskij, Teoria della letteratura 245 ss.). Il suo funzionamento è schematicamente questo: dopo la narrazione di un'azione in cui sono coinvolti due o più personaggi (o dopo l'inizio del romanzo) il narratore segue costantemente uno dei personaggi nel suo allontanamento (tacendo degli altri dopo aver indicato un loro eventuale obbiettivo, o una loro situazione di immobilità coatta), sempre seguendo il primo personaggio finché quegli altri verranno ad incrociare il suo percorso. A questo punto, se il personaggio ritrovato, nel tempo in cui è stato assente dal racconto, ha compiuto qualche azione degna di essere riferita, sarà lui stesso o un testimone a narrarla ad eventuali interessati.

Negli Scherzi di Fortuna, a esempio, l'attenzione è fin dall'inizio centrata su Cleonte, e la narrazione segue il suo incontro con Ramira e la Principessa del Marocco e la sua separazione fino all'approdo a Valenza (N. S. 26 ss.). ${ }^{14}$ Successivamente il racconto abbandona Cleonte e si focalizza sul Principe del Marocco, che giunge ad Ampuria e, invaghitosi della sovrana di quella città, sfida a duello, per futili motivi, un cavaliere sconosciuto, rimandone ucciso. Richiesto di spiegazione dalla duchessa, il cavaliere sconosciuto si svela per Cleonte, e le narra tanto i trascorsi già conosciuti dal lettore ("analessi ripe- 
titiva", per Genette, ovvero anafora narrativa), quanto i fatti accaduti dopo la sua uscita dal racconto primo ("analessi completiva": cfr. Genette, Figure III 96-115).

Se questo procedimento è quasi una norma, più rari sono invece $i$ "salti" tra tracce parallele (ad es. "Or volgiamoci un poco alla Leonilda vera"), sempre in qualche modo motivato da presenze di equivoci o da parallelismi psicologici (ad es.: tormento amoroso di Calloandro / di Leonilda: cfr. C. F. 1.141).

Alla scarsa mobilità in senso spaziale fa riscontro l'estrema sincronicità tra il racconto dell'istanza narrativa principale (extradiegetica) e la "storia", riguardo, ovviamente, all'ordine temporale. Ciò significa che solo molto raramente gli episodi anacronici (soprattutto analettici) vengono narrati nel racconto primo, ${ }^{15}$ mentre più spesso, e sempre per quelli di una certa lunghezza, sono delegati a personaggi-narratori che si occupano di informare uno o più personaggi (e il lettore) delle vicende esterne agli estremi cronologici del racconto primo (se questo inizia in medias res, come tutti i romanzi di Marini), ${ }^{16}$ o accadute contemporaneamente alla sequenza diegetica narrata nel racconto primo ("storie" parallele). La stessa cosa è valida quando si devono riferire vicende di un personaggio precedenti alla sua entrata in scena nel racconto. I narratori dei racconti metadiegetici sono personaggi di vario genere: scrittori, come Aristo, il narratore delle origini della guerra e degli amori di Tigrinda e Poliarte nel Calloandro ("smascherato" e Fedele); più spesso scudieri, come Zefiro nello stesso Calloandro e Carlino negli Scherzi di Fortuna, o anche cavalieri, congiunti dei personaggi dei quali si racconta la vita, ecc. Questi racconti presentano tutti (quasi sempre i più brevi, sempre i più lunghi), un certo numero di caratteristiche:

a) sono plurimotivati: non c'è solo la necessità di informare il lettore e di motivargli il racconto, ma i narratari "intradiegetici" che li ascoltano sono sempre molto interessati alla vicenda e pregano il narratore di esaudire il loro desiderio (ad es. C. F. $1.14 ; 2.221$ );

b) il racconto occupa un tempo morto e serve per ingannare l'attesa o per far trascorrere il tempo di una sosta (ad es. C. F. 2.221; N. S. 272);

c) il narratore è sempre coinvolto personalmente nella vicenda narrata (è "omodiegetico") e fa costante riferimento alla sua presenza testimoniale ${ }^{17}$ (ad es. C. F. 1.32, 50; N. S. 278);

d) dove questa presenza potrebbe risultare inverisimile, o dove l'informazione data sarebbe eccessiva rispetto alle possibilità di chi narra, ${ }^{18}$ si introducono narratori di terzo grado che raccontano personalmente la propria storia 0 quella di cui sono stati testimoni (ad es. C. F. 1.42; N. S. 278);

e) la presenza del narratario, nei racconti molto lunghi, è sottolineata da una sua reazione emotiva: questo evita che a lungo andare il carattere metadiegetico si perda di vista e il racconto divenga "pseudodiegetico" (ad es. C. F. 2.258; N. S. 304).

Talvolta possono aversi complicazioni ingegnose che scompongono o sovrappongono il racconto tra varie istanze narrative, dando un'impressione di 
molteplicità prospettica: così, nel Calloandro, un messaggero racconta a Saladino il racconto di Zefiro a Uranio e Mattamira (cioè Leonilda) sulla fine di Zelim (Calloandro): racconto nel racconto che è anche metaracconto, con narratori e narratari ben presenti e coinvolti emozionalmente. ${ }^{19}$

Passiamo ora ad esaminare sinteticamente due tipi particolari di conformazioni semiotiche, che hanno solitamente occorrenza non trascurabile in qualsiasi narrazione che sia costruita mirando all'unitarietà e alla coerenza tanto della "storia" quanto del "racconto". Si tratta di classi di elementi non sempre tra loro differenziabili per forma e funzienamento. Riprendendo la distinzione barthesiana, potrebbe genericamente dirsi che essi assumano variamente funzioni di nucleo o di catalisi, o si basino sulla confusione di questi due ruoli.

\section{Anafore}

"Con tale termine si indicano ... tutti quei procedimenti diversi di sostituzione che assicurano la chiusura e la coerenza del testo (segni economici in grado di rafforzare l'equivalenza fra termini disgiunti, come pure la coesione e la memorizzazione dell'enunciato)" (Hamon 134) ${ }^{20}$ L'anaforicità, quale tipologia di ridondanza, riguarda classi e sequenze di segni molto diverse, e la sua riscontrabilità, a livello minimale, è praticamente illimitata. All'analisi presente interessano, perché più pertinenti, anafore specificamente narrative, che, non più seguendo Hamon e la Lonzi, proporrei di distinguere in due classi:

a) anafore di "racconto", ovvero ripetizioni o anticipazioni di un evento accaduto una sola volta (racconto "ripetitivo" secondo Genette, Figure III 164-5), introdotte di solito, e segnalate, da un intervento del "discorso" nel racconto ${ }^{21}$ ("abbiamo visto", vedremo che", "non si deve dimenticare che", ecc.); l'istanza narrativa che le pronuncia è in questo caso quella principale (extradiegetica);

b) anafore diegetiche: eventi che, sul piano della "storia", ne richiamano altri già accaduti; oppure: sogni, profezie, progetti, ecc.; o anche: iterazioni di racconto compiute da istanze narrative subordinate (intradiegetiche).

Questo schema ci dà la misura di quanto possa essere ampia la distribuzione e l'occorrenza di tali forme, e quanto interminabile una trattazione esauriente: procederemo dunque ad esaminare, a titolo di esempio, qualche caso particolarmente significativo. Per quanto riguarda le anafore del tipo a), va detto che nei romanzi di Marini (tranne che parzialmente negli Scherzi, dove il narratore si assume particolari funzioni di commento ed interpretazione per favorire la comprensione dell'isotopia religiosa), gli interventi del "discorso" nel racconto sono estremamente rari, e solo poche volte significativi. ${ }^{22} \mathrm{Ne}$ citerò un esempio, tratto dal Calloandro Fedele, avente una funzione ambiguamente prolettica. Esso fa parte di una lunga ed insolita apostrofe del narratore ad un personaggio ("Or dove vai Leonilda? . . . ") che, più che fornire una anticipazione precisa e sicura, si limita ad adombrare un possibile 
narrativo, condizionato in qualche misura dalla "scelta" del personaggio o da una imprevedibile volontà fatale:

$\mathrm{Ma}$, oimè, non iscorgo come tu possa sfuggire le imminenti sciagure, mentre saper non puoi esser vivo il tuo Cavaliere di Cupido, \& essere lo stesso Calloandro. La via, che può condurti a sì fatte cognizioni, è il condescender a queste nozze, cotanto abborrite; oh quanto è ella ardua, s'hai cuore amante, cuor generoso, cuor risentito. (C. F. $1.570-571)$

Previsione, questa, apparentemente ambigua ma, al di là della finzione dell'alternativa, sostanzialmente tautologica e riconfermante un lieto fine sul quale i dubbi del lettore dovrebbero essere ben pochi.

Per le anafore di tipo b), limiterò l'esemplificazione ad una vera e propria costante anaforica che percorre dall'inizio tutto il Calloandro. Si tratta di una catena di anafore (a partire da una "catafora" fondamentale) caratterizzate dal progressivo disambiguamento del messaggio cui si riferiscono, dunque da un'implicazione ricorsiva che dà luogo ad anafore di secondo grado. Il destinatario del messaggio è duplice: il lettore, il personaggio.

All'inizio del romanzo, o meglio della "storia", vi è la profezia del saggio Aristone, raccontata da Aristo in un racconto eziologico sulla guerra tra Grecia ed Asia e l'inimicizia di Poliarte e Tigrinda:

A lei [Leonilda] riserba il fato trionfar dell'Imperio Greco; voi [Tigrinda] vi accingete indarno. Moderate dunque gli impeti dello sdegno, e non v'incresca l'aspettar la maturezza della stagione. E voi, Regina, con le grandezze, che via maggiori vi promette il cielo, sofferite con patienza la dilazione delle vendette contro colui [Poliarte], che più per colpa di destino, che di cuore, mancò di fede. (C. F. 1.60)

La profezia è di per sé ambigua perché non specifica il significato di "trionfar": se vada inteso in senso bellico, o al contrario in senso amoroso e pacifico. L'interpretazione che viene data dai personaggi che ne sono i destinatari è errata semplicemente perché assolutizza il riferimento ad un contesto presente (odio, guerra, vendetta) e non tiene conto del possibile riferimento (questa volta metaforico rispetto al senso proprio militare di "trionfar") ad un contesto futuro (amore tra i nemici, ecc.), che darebbe al messaggio un senso del tutto opposto. D'altra parte la profezia, con il consiglio della "dilazione", produce in un certo senso la successiva vicenda romanzesca. Il vaticinio di Aristone è ricordato più volte (C. $F .1 .238,460,479 ; 2.293)$, nel corso del racconto, dagli stessi personaggi, sia per ribadire l'interpretazione originaria, sia per dubitare della veridicità della stessa profezia, fino a che essa viene "aggiornata" dallo stesso Aristone che, apparso in sogno a Leonilda, la riformula in modo più chiaro per il lettore:

11 fine da lui [il Fato] prescritto alle vostre angosce non è ancor giunto. Non sono per anche cessate le morti, le novelle amare, gli orridi spettacoli, che v'hanno a trafiggere l'anima; havrà triegua allora quando nel vostro seno si estinguerà affatto anche il nome di colui [il Cavaliere di Cupido], la cui morte ora sospirate; e quando da! 
virtuoso amante [Uranio] vi verrà tolto l'Impero di Trabisonda. Allora acquisterete il caro sposo, e 'l nuovo imperio. (C. F. 2.293)

La profezia non viene compresa da Leonilda, che non ha elementi sufficienti per decifrarne l'oscurità. Il lettore, invece, può da parte sua compiere una serie di operazioni interpretative perché ha i termini di riferimento necessari per rendere perspicue le allusioni (ad esempio, "estinguere il nome di colui la cui morte ora sospirate" vuol dire: quando Calloandro si sarà smascherato e smetterà di chiamarsi "Cavaliere di Cupido"). La duplice natura di questo messaggio, più o meno evidente per il lettore, a misura della sua attenzione, ed assolutamente oscuro per il personaggio (il narratario intradiegetico), fa comprendere come tra lettore e personaggio sia notevole il divario nella capacità di "leggere" gli avvenimenti narrati dal testo: su questa differenza si fonda il compiacimento del lettore per la sua abilità previsionale, quel piacere dovuto all'apparente magnificazione delle sue capacità interpretative, all'impressione di possedere l'assoluto dominio sul testo. Se, infatti, "la particolarità che il sistema anaforico non sia soggetto a perturbazione rappresenta un importante fattore della leggibilità di un enunciato" (Hamon 134), l'errore e l'equivoco dipendono proprio dalle perturbazioni che, per il personaggio (narratario delle profezie e dei racconti nel racconto) questo sistema subisce. I malintesi sono così generati dalla non corrispondenza delle anafore (rapporto previsione-evento; non fissità dei nomi e degli attributi dei personaggi, ecc.), dovuta alla conoscenza solo parziale, e diversa da personaggio a personaggio, della loro catena, e dunque alla insufficiente ridondanza di queste disseminazioni semiotiche. Il sistema della leggibilità contiene anche quello della non-leggibilità. Nel primo caso, per il lettore, serve alla coerenza del testo; nel secondo, permette l'esistenza del testo.

\section{Motivazione}

Può un'opera che voglia presentarsi unitaria e coerente essere demotivata? ${ }^{23}$ Se si tratta di un testo narrativo, certamente no. La scelta da parte dell'autore si presentava piuttosto ristretta, venendo a mancare per il rifiuto dell' $h$ istoria una possibilità di motivazione di tipo "realistico" (Tomaševskij, "La costruzione dell'intreccio" 328-33), e parimenti escludendo, per la scarsa importanza accordata al rispetto del verisimile, la più "economica" delle motivazioni, la più semplice e naturale, la meno costosa in termini semiotici (Genette, Figure II 68). Si pone dunque un problema di scelte sostitutive, mentre d'altra parte il privilegio accordato all'universo diegetico comporta la rinuncia, perlomeno per Calloandro e Gare, ad un continuo intervento digressivo del narratore nelle forme del "discorso", ed esclude la presenza di una mediazione interpretativa inscritta nel testo e separata dalla narrazione in quanto pregiudiziale per la compattezza del racconto.

Da parte sua, la motivazione può apparire essenzialmente in due forme: in modo puntuale, giustificando ogni singolo evento, o ciascuna microse- 
quenza causa-effetto; ed in modo sequenziale, rendendo plausibile l'intero svolgimento delle peripezie, e lo scioglimento del romanzo.

Una motivazione di questo secondo tipo, che non coincida con quella realistica ("è successo così e non ha senso dare spiegazione") o col verisimile ("potrebbe essere successo così, anzi non c'è bisogno di spiegazione perché tutto è molto probabile"), deve necessariamente avere una sua propria coerenza interna, ed essere dotata nel suo insieme di significato e di referente (la natura psichica dell'uomo, le leggi biologiche, la volontà di Dio, ecc.). Indubbiamente la "Fortuna", diversamente dal puro caso (che è una non-motivazione), gode di un certo privilegio di motivazione qualora venga presentata in forma quasi personificata, e dotata di attributi (incostanza, malvagità, astuzia). ${ }^{24}$ Tuttavia la sua azione è, nella norma, di tipo puntuale, e di portata limitata. La ripetizione continua, ad ogni evento, della funzione della Fortuna come causa può solo avere l'effetto di magnificare il senso di immotivazione. Un primo grado di strutturazione coerente degli accidenti di Fortuna è il Destino, che però riguarda i personaggi solo singolarmente, sopperendo tuttavia per qualcuno (ad esempio Calloandro) all'interiore demotivazione della condotta. La concordanza, l'intreccio, la collisione di destini, potrebbe essere, pessimisticamente, non spiegata ${ }^{25}$ (e ciò apparirebbe in una forma a suo modo "verisimile": tutto, nella realtà, è caso o destino, e comunque inconoscibile), ma il romanzo a lieto fine non può ricorrere a tale ideologia.

Certamente la struttura più adatta per dare senso a caso, fortuna, destino, è la presenza di un disegno provvidenziale. Non si tratta di Provvidenza necessariamente cristiana (il modello senechiano e stoico non era certo sconosciuto al Seicento): per quanto più volte nominata, nel Marini pagano del Calloandro e delle Gare essa si riduce a ben poca cosa, invocata dove la "stravaganza degli accidenti" è davvero insostenibile (C. F. 1.376, 440, 463). Diverso è il caso degli Scherzi, romanzo ambientato in una guerra tra Cristiani e Musulmani, dove lo stesso tipico sistema di coincidenze avventurate svela il disegno provvidenziale, e dove dunque gli stessi classici procedimenti narrativi romanzeschi sono magnificati e risemantizzati in senso religioso, divenendo veicolo per il proposito superiore. Comunque il richiamo alla Fortuna ad ogni occasione non può portare un grande contributo di motivazione. D'altronde non v'è nessuna ragione per escludere che Marini fosse non interessato alla motivazione razionale a tutti i costi, e preferisse quella che, pur non recando nessuna giustificazione, contribuisse a fornire un'immagine di coerenza lasciando immotivate o addirittura amplificate la stravaganza e l'improbabilità. Questo tipo di motivazione, che è in effetti una pseudomotivazione, può avere una grande varietà di forme, e operare a livelli semiotici e gradi di efficacia diversissimi. Può assumere ad esempio l'apparenza di una motivazione "forte", e fingere un rapporto causa-effetto: è questo il caso della somiglianza di Calloandro e Leonilda, presagio del loro destino e condizione necessitante la loro unione (C. F. 2.101-2).

Altre volte non sono in gioco rapporti causali espressi come tali, e la mo- 
tivazione assume la forma di un procedimento propriamente anaforico. Si può esaminare un esempio tratto dagli Scherzi di Fortuna, dove tali espedienti hanno una frequenza altissima: il duca Ernesto racconta a Cleonte che ha scelto di ritirarsi in romitaggio per la morte della moglie Elisa, avvenuta verisimilmente per annegamento dopo uno strano incidente (N. S. 136-39); il suo predecessore nell'eremo, poco prima di morire, gli aveva espresso la profezia che avrebbe presto ritrovato sua moglie, messaggio che Ernesto interpreta come annuncio della prossimità della sua morte; finito il racconto, e partito Cleonte, Ernesto ripensa con dolore alla perdita della moglie, ed ecco che vede, da una nave naufragata, avvicinarsi disperatamente alla riva un uomo e una donna: l'uomo annega, la donna è salvata da Ernesto: è Elisa (148-52). La sequenza, dunque, ricca di molte simmetrie (il replicato pericolo di morte per acqua; la morte dell'uomo che nuotava con lei, suo ostinato pretendente musulmano, mentre ritrova il vero e cristiano marito), ci mostra una successione di discorsi ed eventi (1: ricordo della profezia; 2: evocazione del personaggio; 3: sua apparizione), in serie evidentemente anaforica, recante una parvenza di motivazione dove la ridondanza colma l'assenza di qualsiasi rapporto causale (la profezia, del resto, è male interpretata, e il suo richiamo nella narrazione a Cleonte non è comunque motivante rispetto all'evento-apparizione). Ovviamente, tutta la vicenda va letta come atto della volontà di Dio, ma il procedimento anaforico serve comunque a stabilire una continuità altrimenti mancante e a fungere così da sostituto narrativo, più che da semplice fenomeno, di un deus post machinam che non può apparire nel racconto.

Altro motivo frequentissimo è il seguente: un personaggio sente occulta ammirazione o amore per un altro personaggio che in seguito si scoprirà essere (o che diventerà) suo congiunto; viceversa prova disprezzo per un parente presunto che in seguito si scoprirà non essere tale (C. F. 2.111; 3.12, 106, $196 ; N . G .109$ ). Si ha in questo modo un rapporto in ordine inverso rispetto al normale: l'effetto precede e presagisce la causa, ovvero la conoscenza dello stato delle cose. Questi occulti ed inesplicabili sentimenti possono evitare l'infrazione di interdetti (incesti, violenze verso i congiunti) o permettere gesti d'affetto altrimenti immotivati (N. G. 66-77).

Difficilmente però si può dare una descrizione singolarmente esaustiva del funzionamento di tali procedimenti, apparendo essi raramente isolati e più spesso arricchiti e coadiuvati da vari elementi indiziali. Essi non sono del resto vere motivazioni, ma parvenze che si accumulano, si sovrappongono, si confondono e senza giustificare peraltro la ratio del mondo narrato riempiono i vuoti di un'incombente discontinuità, generando un'attesa che non sarà delusa. Del resto, scrive Barthes in una pagina celebre, "tutto lascia pensare che . . . la molla dell'attività narrativa sia proprio la confusione fra consecutività e consequenzialità, in quanto ciò che viene poi è letto come causato da: il racconto sarebbe, in questo caso, un'applicazione sistematica dell'errore logico denunciato dalla scolastica post hoc ergo propter hoc, che 
potrebbe ben essere l'emblema del Destino, di cui il racconto non è altro che la lingua ..." $(20)^{26}$

\section{University of Toronto}

\section{NOTE}

1 Per un quadro attento ed esauriente delle polemiche e dei giudizi sul romanzo, cfr. Mancini 1981, 65 ss. c passim.

2 Un panorama dettagliato degli interessi tematici e stilistici di vari autori, con riguardo particolare al valore delle prefazioni, si trova in Mancini 1981 (segnatamente, per G. A. Marini, alle pp. 79-90).

3 Di ciascuno dei suoi tre romanzi Marini produsse stesure diverse, emendando ed accrescendo i testi di partenza. Per il Calloandro, la prima versione, detta "sconosciuta" perché presentata come opera di Gio. Maria Indris Bocmo (anagramma imperfetto del nome di Marini), tradotta dal tedesco da Giramo Bisii Romano (ancora un anagramma), fu stampata a Bracciano presso Fei nel 1640 e completata della seconda parte a Genova, da Calenzani, nel 1641. La seconda stesura, corretta in più punti, detta Calloandro ... smascherato perché vi compariva il nome di Marini, uscì sempre nel 1641 a Genova dallo stesso Calenzani. Nel 1653 apparve infine a Roma, da Corvi, la terza e definitiva versione, contenente importanti innovazioni: il protagonista non tradiva più l'adorata Lconilda e diventava così un Calloandro Fedele. Le Gare de' disperati, pubblicate a Bologna da Zenero nel 1644 ebbero una nuova stesura emendata e complicata nella trama (Le nuove gare. . . , Genova, Guasco, 1653; stessa cosa accadde per gli Scherzi di Fortuna a pro' dell'Innocenza (Genova, Calenzani, 1662), rimaneggiati e ristampati nel 1666 presso lo stesso editore (I nuovi scherzi . . . ). Per le citazioni dai testi e dalle prefazioni ci si è serviti delle edizioni elencate in bibliografia. Dal Calloandro Fedele e dalle Gare Marini stesso trasse due tragicommedie omonime, pubblicate a Genova rispettivamente da Guasco nel 1656, e da Calenzani nel 1660, su cui cfr. Conrieri 1987. Della grande fortuna dei romanzi di Marini presso il pubblico in Italia e nel resto d'Europa fa fede il numero enorme di traduzioni e ristampe: esse sono state diligentemente classificate e annotate da Mancini nei due saggi di bibliografia da lui dedicati al romanzo secentesco (1970-1 e 1975). Sulla complessa vicenda redazionale ed editoriale del Calloandro cfr. Raimondi (99-118) e Conrieri 1970 (ora parzialmente rifuso in Conrieri 1974). Sui significati e le implicazioni dell'opera di rifacimento dei testi da parte di Marini, cfr. Ortolani e Albani. Per l'ammirazione ed il plauso di un arguto contemporaneo, si veda il giudizio dedicato al Calloandro da Francesco Fulvio Frugoni nei "Quinti latrati" del suo Cane di Diogene (282-3).

4 Si scrivevano addirittura "antiche historie del Nuovo Mondo" (Tomasi). Significativa e poIemica la posizionc espressa da Luca Assarino nella premessa alla sua Almerinda: "emmi paruto meglio il favoleggiare sulle Historie che historiare sulle Favole".

5 Nelle stampe dei romanzi di Marini qui utilizzate, le pagine prefatorie non sono numerate. Le edizioni, con le relative sigle, sono indicate nell'elenco delle opere citate.

6 Cfr. Huet: "la verisimiglianza, che non si trova sempre nella Storia, è essenzialc nel Romanzo" (6-7). Essa deve dunque coincidere, secondo la classica dottrina francese, col dover essere, con la misura ideale delle cose (Genctte, Figure II 43-69). Sul problema del discredito del verisimile in Italia come portato della crisi dell'inventio poetica, cfr. Morpurgo Tagliabue 119-97.

7 A proposito del Calloandro, Frugoni rileva che $\mathrm{i}$ "racconti" contenuti nel romanzo "non acquistano fede col verisimile" (282).

8 Cfr. C. Sc., "a' lettori": "da esso oltre il diletto tu possa anche utilmente raccogliere quale esser debba un vero amante, un vero amico, un Prencipe, un Eroe, un Cavaliere, un fedel servo". 
9 Lo studio di Fantuzzi su Biondi e Brusoni e quello di Getto su "ll romanzo veneto nell'età barocca" hanno restituito l'immagine di testi narrativi in cui la trama è dispersa in serie di azioni promosse dagli spostamenti di vari eroi seguiti in narrazioni parallele. Si tratta, come è evidente, di una vistosa eredità strutturale del romance cavalleresco, talvolta risolta in modo maldestro con tecniche di entralacement piuttosto approssimative. Non sono però d'accordo con Fantuzzi sulle premesse generalizzanti del suo lavoro: i modelli e i meccanismi narrativi additati sono validi per le opere esaminate ma non sono estensibili per ragioni teoriche (lo scarso valore ermeneutico dell'uso dei patterns funzionali vetero-formalisti, ad esempio), tanto quanto per la varietà obbiettiva dei testi, ad altri prodotti dello stesso genere ed epoca letteraria.

10 L'ultima ristampa accertata delle $G$. si ebbe a Bracciano nel 1799. Per il C. F. si arriva addirittura ad un'edizione padovana del 1887 (editore Piazza; cfr. Mancini 1970-1). II nome stesso di Calloandro assurse a dignità antonomastica, pur nel discredito e nella caricatura, per indicare il tipo dell'amante verboso e dalle passioni esagerate: si veda ad esempio la goldoniana Vedova scaltra (III.7); cfr. anche Le virtuose ridicole (1II.6).

11 La trattazione dei meccanismi miranti all'unità non potrà tuttavia tener sempre distinti i due livelli della narrativa ("storia" e "racconto", o, tradizionalmente, fabula e intreccio). Sono infatti in gioco procedimenti che investono entrambi i piani operando in modo interdipendente. Banale è l'esempio seguente: per evitare alla voce narrante principale la discontinuità di un salto cronologico, permettendo però al racconto di iniziarsi in medias res, è necessario che si introducano narratori delegati e narratari opportunamente curiosi e interessati, e questa è un'operazione che riguarda la fabula (l'inventio, mentre il cambio di voce pertiene all'intreccio).

12 Intendo l'espressione "autore"-narratore nel senso usato da Chatman (55). Si identifica con essa il narratore extradiegetico: se c'è bisogno, per Chatman, di un "contesto pragmatico ... che connetta il parlante con lui stesso" (l'autore), tale contesto è nel nostro caso proprio nei discorsi di prefazione.

13 Restano ovviamente esclusi i personaggi che appaiono nei racconti esplicativi ed analettici in genere.

14 Molto significativa per l'uso di questa tecnica è la sequenza in $C$. $F .1 .263$ ss.

15 Intendiamo per "racconto primo" la narrazione principale a voce del narratore extradiegetico (l'“autore"-narratore).

$16 \mathrm{Nel}$ Calloandro, l'inizio in medias res con integrazione a voce di personaggio-narratore è solo in $C$. Sm. e C. F. . Negli Scherzi di Fortuna l'integrazione analettica principale è solo in $N$. S., di cui costituisce l'intero cap. 6 .

17 Cfr. Genette, Figure III: "I'orientazione del narratore verso se stesso . . . determina una funzione con omologie spiccatissime nei confronti di quella chiamata da Jakobson, un po' impropriamente, 'funzione emotiva': è la funzione che informa sulla parte presa dal narratore, in quanto tale, alla storia da lui narrata, cioè sul rapporto tra narratore e storia: rapporto affettivo, certo, ma anche morale o intellettuale, e che può prendere la forma di una semplice testimonianza, come quando il narratore indica la fonte da cui deriva la sua narrazione, $o$ il grado di precisione dei suoi ricordi personali, o i sentimenti risvegliati in lui da un certo episodio: abbiamo in questo caso qualcosa che potrebbe essere chiamato funzione testimoniale o di attestazione" (304).

18 Si avrebbe in questo caso un 'infrazione delle norme del codice che Genette denomina "parallessi" (Figure III 242-4, e in relazione più specifica alla nostra tipologia, Nuovo discorso del racconto 66$)$.

19 Intendo "metaracconto" come racconto di o su un racconto, discostandomi dall'uso proposto da Genette (Figure III 276), troppo lontano dal senso abituale del prefisso meta anche in narratologia (Prince 174-94). Uso invece "metadiegetico" nel senso di Genette (Figure III 276), cioè come "racconto nel racconto" ovvero racconto di secondo grado. Per la nozione di voce il riferimento è, ovviamente, ancora a Genette (Figure III 259-310). 
20 L'applicazione all'analisi del racconto del termine retorico si deve alla Lonzi e a Maillard. Hamon, sulla loro scorta, preferisce distinguere tra "anaforici (segni che rinviano ad uno anteriore dello stesso enunciato) e cataforici (segni che rinviano ad uno ulteriore dello stesso enunciato)" (134). Preferiamo tuttavia non complicare inutilmente la terminologia e usare indistintamente "anafora". Cfr. anche Prince 212.

21 Intendo "discorso" come intervento del locutore segnalato da indizi di tipo pronominale (io, $t u$, deittici) e da certi tempi verbali (tempi dipendenti dal presente): il riferimento è a Benveniste 230 ss. e a Rousset; cfr. anche Genette Figure II 23-41.

22 Mancano dunque, o vi appaiono molto raramente, appelli al lettore, digressioni commentative, o addirittura, "metalessi", ed altre forme molto diffuse presso altri romanzieri; si veda a titolo d'esempio la prefazione a La Vergine Parigina di Frugoni. Si veda pure quanto scrive Getto, a proposito dei romanzieri veneti, sul ricorrente "affacciarsi alla ribalta dell'autore" (324-5).

23 Uso il concetto di "motivazione" nel senso tradizionale proposto da Tomaševskij ("La costruzione dell'intreccio" 326-37) e Šklovskij 287 e passim. Per ritornare ancora nell'ambito dell'anafora, l'uso di sogni e profezie, come espedienti per "motivare", dall'inizio o in qualsiasi punto del racconto, lo scioglimento finale, ha ovviamente origine nell'archetipo stesso del romanzo occidentale, ovvero nel romanzo ellenistico. Huet condannava l'uso che Eliodoro di Emesa ne aveva fatto nelle Etiopiche, parlando spregiativamente di macchine. Eliodoro, pur non usando le "macchine" per sciogliere l'intrigo, ne avrebbe inopportunamente abbondato nel resto del romanzo (24). "Motivati" in senso linguistico sono i nomi di alcuni personaggi, tanto singolarmente (Calloandro, Altobello, Stella in $C$. $F$; Fidalma in $N$. G. e molti altri), quanto in coppie genetiche (Leonilda-Tigrinda, Orgoglione-Branditone in $C$. $F$.) o d'amanti (Spina-Spinamante in N. G.) o addirittura con riferimento intertestuale (Spinalba in $C . F$. la cui vicenda ha molte affinità con quella dell'ariostesca Fiordispina).

24 In un'operina devota dello stesso Marini, ll caso non a caso, dove sugli accidenti humani s'impara ad intendere il linguaggio divino, Caso e Fortuna sono entrambi personificati ("due gran personaggi") e, nella svalutazione della loro sostanzialità (a favore della Provvidenza), di fatto assimilati. Però è indubbio che nel romanzo sia la sola Fortuna a godere, tradizionalmente, di attributi figurativi (la ruota, ecc.).

25 Sull'uso giustificazionistico della fortuna, e per gli atteggiamenti fatalistici di altri romanzieri, cfr. Fantuzzi 230-1. Cfr. anche gli studi di Danicla Dalla Valle (1968 e 1970).

26 Prendendo in esame un ultimo "procedimento per l'unità", non posso dirmi d'accordo con l'Albani secondo cui "en accentuant les symetries" della trama nell'ultima stesura del Calloandro ( $\mathrm{ma}$ il discorso dovrebbe farsi anche per $N$. G.) Marini otterrebbe una maggiore "unité d'action" (268). Questo tipo di simmetria è tutt'altro che favorevole all'unità d'azione in sé e per sé. A ricevere connotazioni unitarie, ma ad un livello di percezione metanarrativa di ordine superiore, è semmai il procedimento inventivo dell'autore. Bisogna ricordare infine la definizione suggestiva ma esagerata (per l'assenza, come s'è messo in luce, di molte caratteristiche primarie) di "roman classique" proposta da Gardair.

\section{OPERE CITATE}

Albani, Hélène, "Evolution de thèmes et de structures dans l'oeuvre romanesque de G. A. Marini". Rev'ue des études italiennes 15 (1969): 258-78.

Assarino, Luca. L'Almerinda. Venezia: Sarzina, 1640.

Barthes, Roland. "Introduzione all'analisi strutturale dei racconti". L'analisi del racconto. Milano: Bompiani, 1969.

Benveniste, Émile. Problemi di linguistica generale. Milano: Il Saggiatore, 1971.

Chatman, Scymour. Storia e discorso. La struttura narrativa nel romanzo e nel film. Parma: Pratiche, 1981.

Conrieri, Davide. "Il Calloandro Fedele di Giovan Ambrosio Marini". Giornale storico della letteratura italiana 147 (1970): 260-91. 
"Il romanzo ligure nell'età barocca". Annali della Scuola Normale Superiore di Pisa. Classe di lettere e filosofia 3.4 .3 (1974): 925-1139.

"La rielaborazione teatrale di romanzi nel Seicento: considerazioni e prime indagini”. Sul romanzo secentesco. Ed. Gino Rizzo. Galatina: Congedo, 1987. 29-100.

Dalla Valle, Daniela. "ll tema della fortuna nella tragedia italiana rinascimentale e barocca". Italica 44 (1968): 180-208.

La frattura. Studi sul barocco letterario francese. Ravenna: Longo, 1970.

Fantuzzi, Marco. Meccanismi narrativi del romanzo barocco. Padova: Antenore, 1975.

Frugoni, Francesco Fulvio. Il Cane di Diogene. Venezia: Bosio, 1689.

La Vergine Parigina. Venezia: Combi e la Nou, 1669.

Gardair, Jean Michel. "Trois romans baroques italiennes". Revue des études italiennes 13 (1967): 105-47.

Genette, Gerard. Figure II. Torino: Einaudi, 1972.

Figure III. Torino: Einaudi, 1976.

Nuovo discorso del racconto. Torino: Einaudi, 1987.

Getto, Giovanni. Barocco in prosa e in poesia. Milano: Rizzoli, 1969.

Hamon, Philip. Semiologia, lessico, leggibilità del testo narrativo. Parma-Lucca: Pratiche, 1977.

Huet, Pierre Daniel. Trattato sull'origine dei romanzi. Torino: Einaudi, 1977.

Lonzi, Lidia. “Anaphore et récit”. Communications 17 (1970): 133-42.

Maillard, M. “Anaphore et cataphores”. Communications 19 (1972): 93-104.

Mancini, Albert N. Romanzi e romanzieri del Seicento. Napoli: S.E.N., 1981.

"Il romanzo nel Seicento. Saggio di bibliografia". Studi Secenteschi 11 (1970):

205-74; 12 (1971): 443-98.

"Il romanzo italiano del Seicento. Saggio di bibliografia delle traduzioni in

lingua straniera". Studi Secenteschi 16 (1975): 183-217.

Manzini, Giovan Battista. Il Cretideo. Bologna: Ferroni, 1637.

Marini, Giovan Ambrosio. Il Calloandro di Gio. Maria Indris Bocmo traslato di tedesco in italiano da Giramo Bisii Romano (= C. Sc.). Venezia: Turrini, 1641.

Il Calloandro, altra volta di Gio. Maria Indris Boemo: hora Gio. Ambrosio

Marini smascherato (=C.Sm.). Venezia: Turrini, 1652.

Il Calloandro Fedele (= C. F.). Roma: Corvi, 1653.

Le gare de' disperati $(=$ G.). Venezia: Turrini, 1652.

Le nuove gare de' disperati (= N. G.). Genova: Vincenti, 1665.

Scherzi di fortuna a pro' dell 'innocenza (= S.). Genova: Calenzani, 1662.

Nuovi scherzi di fortuna a pro' dell 'innocenza $(=N$. S.). Venezia: Conzatti, 1678.

Il caso non a caso, dove sugli accidenti humani s'impara ad intendere il linguag-

gio divino. Genova: Calenzani, 1650.

Morpurgo Tagliabue, Guido. "Aristotelismo e barocco". Retorica e barocco. Ed. E. Castelli. Roma: Bocca, 1954. 119-96.

Ortolani, Donata. “Gio. Ambrogio de' Marini”. Rassegna della letteratura italiana 73 (1969): 402-20.

Prince, Gerald. Narratologia. Parma: Pratiche, 1984.

Raimondi, Ezio. Anatomie secentesche. Pisa: Nistri-Lischi, 1966.

Rousset, Jean. "La prima persona del romanzo". Strumenti critici 6 (1972): 259-74.

Šklovskij, Victor Borisovic. Teoria della prosa. Torino: Einaudi, 1976.

Tomaševskij, Boris. "La costruzione dell'intreccio". I formalisti russi. Ed. Tz. Todorov. Torino:

Einaudi, 1968. 326-37.

Teoria della letteratura. Milano: Feltrinelli, 1978.

Tomasi, Tomaso. La Spinalba. Antica historia del Nuovo Mondo. Venezia: Valvasense, 1647. 